\title{
SOME MAXIMUM PRINCIPLES IN SEMILINEAR ELLIPTIC EQUATIONS
}

\author{
PHILIP W. SCHAEFER
}

\begin{abstract}
We develop maximum principles for functions defined on the solutions to a class of semilinear, second order, uniformly elliptic partial differential equations. These principles are related to recent theorems of Protter and Protter and Weinberger and to a technique initiated by Payne for the determination of gradient bounds on the solution of the equation.
\end{abstract}

1. Introduction. In [3] Payne introduced a technique, which utilizes a maximum principle for a function defined on solutions to an elliptic partial differential equation, in order to obtain bounds for the gradient of the solution of the relevent differential equation. Several authors have contributed to the growing literature developing this technique. In their work (see the references cited here, especially [7], and the references therein), the authors seek estimates on the solution, the gradient of the solution, or other quantities of physical importance and/or extend the method to more general elliptic or parabolic differential equations. Early in the development of this method, the results were obtained when the principal part of the elliptic equation was the Laplace operator.

Sperb [6] was the first to extend the results of Payne to a second order uniformly elliptic equation of the form

$$
\left(a^{i j}(x) u_{, j}\right)_{, i}+b^{i}(x) u_{, i}+c(x) f(u)=0,
$$

where the comma notation $u_{, k}$ signifies partial differentiation with respect to the $k$ th coordinate variable and the repeated index in a single term denotes summation over that index from 1 to $n$. Sperb's extension depended heavily on differential geometric quantities and the Riemannian metric $g^{i j}$ induced by the principal part of (1.1), on the coefficients in (1.1), and the geometry of the domain.

Sperb's development was greatly simplified by Protter [5] for general second order uniformly elliptic equations of the form

$$
a^{i j}(x) u_{, i j}+b^{i}(x) u_{, i}+f(u)=0,
$$

where by uniformly elliptic, we mean, the symmetric matrix $\left(a^{i j}\right)$ is positive definite and satisfies the uniform ellipticity condition

$$
a^{i j}(x) \xi_{i} \xi_{j} \geq \lambda|\xi|^{2}, \quad x \in \Omega, \xi \in R^{n},
$$

for some positive constant $\lambda$. Protter showed that a certain function defined on solutions to (1.2) attains its maximum either on the boundary of the domain $\Omega$ or at a critical point of the solution, i.e., where $\operatorname{grad} u=0$.

Received by the editors August 6, 1985.

1980 Mathematics Subject Classification. Primary 35B50, 35J15.

Key words and phrases. Semilinear elliptic equations, maximum principles. 
In this work we use an inequality from [4] to obtain maximum principles for functions defined on the solutions to uniformly elliptic equations of the form

$$
a^{i j}(x) u_{, i j}+b^{i}(x) u_{, i}-c(x) f(u)=0 .
$$

We obtain principles in $\S 2$ which state that the maximum of the function cannot be attained in the interior of $\Omega$ unless it is a constant and thus do not encounter the possibility of an occurrence at a critical point in the domain. In $\S 3$ we briefly illustrate the application of these principles for the determination of solution or gradient estimates.

2. Results. Let $\Omega$ be a bounded domain in $R^{n}, n \geq 2$, and let $u$ be a $C^{3}$ solution of the uniformly elliptic equation (1.4) in $\Omega$.

We define the function

$$
P(x)=\frac{u_{, k} u_{, k}}{c(x)}+\gamma \int_{0}^{u} f(t) d t
$$

where $\gamma$ is a positive constant to be determined and $c(x) \geq c_{0}>0$. By a straightforward computation, we have

$$
\begin{gathered}
P_{, i}=2 c^{-1} u_{, k} u_{, k i}-c^{-2} u_{, k} u_{, k} c_{, i}+\gamma f(u) u_{, i}, \\
P_{, i j}=2 c^{-1} u_{, k j} u_{, k i}+2 c^{-1} u_{, k} u_{, k i j}-2 c^{-2} u_{, k} u_{, k i} c_{, j}-2 c^{-2} u_{, k} u_{, k j} c_{, i} \\
-c^{-2} u_{, k} u_{, k} c_{, i j}+2 c^{-3} u_{, k} u_{, k} c_{, i} c_{, j}+\gamma f^{\prime} u_{, j} u_{, i}+\gamma f u_{, i j},
\end{gathered}
$$

where $f^{\prime}$ denotes differentiation with respect to $u$. We now write

$$
\begin{aligned}
L P \equiv & a^{i j} P_{, i j}+b^{i} P_{, i} \\
= & 2 c^{-1} a^{i j} u_{, k j} u_{, k i}+2 c^{-1} a^{i j} u_{, k} u_{, k i j}-2 c^{-2} a^{i j} u_{, k} u_{, k i} c_{, j} \\
& -2 c^{-2} a^{i j} u_{, k} u_{, k j} c_{, i}-c^{-2} a^{i j} u_{, k} u_{, k} c_{, i j}+2 c^{-3} a^{i j} u_{, k} u_{, k} c_{, i} c_{, j} \\
& +\gamma f^{\prime} a^{i j} u_{, j} u_{, i}+\gamma f a^{i j} u_{, i j}+2 c^{-1} b^{i} u_{, k} u_{, k i} \\
& -c^{-2} b^{i} u_{, k} u_{, k} c_{, i}+\gamma f b^{i} u_{, i} .
\end{aligned}
$$

We seek to make the right-hand side of (2.2) nonnegative. To handle the second and ninth terms, we differentiate (1.4) with respect to $x_{k}$, multiply by $2 c^{-1} u, k$, and then solve for these terms. Hence we have

$$
\begin{aligned}
& 2 c^{-1} a^{i j} u_{, k} u_{, k i j}+2 c^{-1} b^{i} u_{, k} u_{, k i} \\
& \quad=-2 c^{-1} a_{, k}^{i j} u_{, k} u_{, i j}-2 c^{-1} b_{, k}^{i} u_{, k} u_{, i}+2 c^{-1} f u_{, k} c_{, k}+2 f^{\prime} u_{, k} u_{, k} .
\end{aligned}
$$

We substitute (2.3) into (2.2) and combine appropriate terms to obtain

$$
\begin{aligned}
L P= & 2 c^{-1} a^{i j} u_{, k j} u_{, k i}-2 c^{-1} a_{, k}^{i j} u_{, k} u_{, i j}-2 c^{-1} b_{, k}^{i} u_{, k} u_{, i} \\
& +2 c^{-1} f u_{, k} c_{, k}+2 f^{\prime} u_{, k} u_{, k}-4 c^{-2} a^{i j} u_{, k} u_{, k i} c_{, j}-c^{-2} u_{, k} u_{, k} L c \\
& +2 c^{-3} u_{, k} u_{, k} a^{i j} c_{, i} c_{, j}+\gamma f^{\prime} a^{i j} u_{, j} u_{, i}+\gamma c f^{2} .
\end{aligned}
$$

We shall now combine the fourth and tenth terms on the right side of (2.4) by

$$
\begin{aligned}
(2.5) \gamma c f^{2}+ & 2 c^{-1} f u_{, k} c_{, k} \\
& =(\gamma-1) c f^{2}+c^{-1}\left[c^{2} f^{2}+2 f u_{, k} c_{, k}+\left(c^{-1} u_{, k} c c_{, k}\right)^{2}-\left(c^{-1} u_{, k} c_{, k}\right)^{2}\right] \\
& \geq(\gamma-1) c f^{2}+c^{-1}\left[c f+c^{-1} u_{, k} c_{, k}\right]^{2}-c^{-3}|\nabla c|^{2} u_{, k} u_{, k} .
\end{aligned}
$$


To combine the first, second, and sixth terms on the right side of (2.4), we employ an inequality from $[4]$. Let $\left(A^{i j}\right)$ be the matrix which is the inverse of the positive definite $\left(a^{i j}\right)$ and let $\left(s^{p k}\right)$ be an arbitrary $n \times n$ matrix. Then since

$$
a^{i j}\left(u_{, i k}+\frac{1}{2} A^{i p} s^{p k}\right)\left(u_{, j k}+\frac{1}{2} A^{j q} s^{q k}\right) \geq 0
$$

one has the inequality

$$
s^{k i} u_{, k i} \geq-a^{i j} u_{, k j} u_{, k i}-\frac{1}{4} A^{p q} s^{p k} s^{q k}
$$

for the arbitrary matrix $\left(s^{k i}\right)$. Consequently we have

$$
\begin{aligned}
2 c^{-1} & a^{i j} u_{, k j} u_{, k i}-2 c^{-1} a_{, j}^{k i} u_{, j} u_{, k i}-4 c^{-2} a^{i j} c_{, j} u_{, k} u_{, k i} \\
& =2 c^{-1}\left\{a^{i j} u_{, k j}-a_{, j}^{k i} u_{, j}-2 c^{-1} a^{i j} c_{, j} u_{, k}\right\} u_{, k i} \\
& \geq-(2 c)^{-1} A^{p q} s^{p k} s^{q k}
\end{aligned}
$$

where we have chosen

$$
s^{k i}=-a_{, j}^{k i} u_{, j}-2 c^{-1} a^{i j} c_{, j} u, k .
$$

We finally substitute (2.5) and (2.6) into the right side of (2.4) and rearrange terms so that

$$
\begin{aligned}
L P \geq\{ & \gamma f^{\prime} a^{i j}+\left(2 f^{\prime}-c^{-2} L c-c^{-3}|\nabla c|^{2}\right) \delta^{i j}-2 c^{-1} b_{, j}^{i} \\
& -(2 c)^{-1}\left[A^{p q} a_{, j}^{p k} a_{, i}^{q k}+2 c^{-1} A^{p i} a_{, j}^{p k} a^{k m} c_{, m}+2 c^{-1} A^{j q} a^{k l} c_{, l} a_{, i}^{q k}\right. \\
& \left.\left.+4 c^{-2} A^{j i} a^{k l} c_{, l} a^{k m} c_{, m}\right]\right\} u_{, j} u_{, i} \\
& +2 c^{-3} u_{, k} u_{, k} a^{i j} c_{, i} c_{, j}+(\gamma-1) c f^{2}+c^{-1}\left[c f+c^{-1} u_{, k} c_{, k}\right]^{2},
\end{aligned}
$$

where $\delta^{i j}$ is the Kronecker delta. Now for $\gamma$ sufficiently large the right side can be made nonnegative as desired. We thus have the following.

THEOREM 1. If $u$ is a $C^{3}$ solution of (1.4), where the coefficients $a^{i j}, b^{i} \in$ $C^{1}(\bar{\Omega}), c \in C^{2}(\bar{\Omega})$ and $c(x) \geq c_{0}>0$ and the function $f \in C^{1}(R)$ has a derivative which is bounded below by $f^{\prime}(u) \geq \alpha>0$, then there exists a positive constant $\gamma$ sufficiently large $(\delta \geq 1)$ such that

$$
P(x)=\frac{u_{, k} u_{, k}}{c(x)}+\gamma \int_{0}^{u} f(t) d t
$$

cannot attain its maximum value in $\Omega$ unless it is a constant.

As in [5], one would like to weaken the requirement that the derivative of $f$ have a positive lower bound. Before doing such, we note that by an entirely similar analysis as in Theorem 1, motivated by the function introduced in [2], one can deduce

THEOREM 2. If $u$ is a $C^{3}$ solution of

$$
a^{i j}(x) u_{, i j}+b^{i}(x) u_{, i}+c(x) f(u)=0,
$$

where the coefficients $a^{i j}, b^{i} \in C^{1}(\bar{\Omega}), c \in C^{2}(\bar{\Omega})$ and $c(x) \geq c_{0}>0$ and the function $f \in C^{1}(R)$ is such that $u f(u) \leq 0$ and $f^{\prime}$ is bounded above, then there exists a positive constant $\gamma$ sufficiently large such that

$$
Q(x)=\frac{u, k u, k}{c(x)}+\gamma u^{2}-2 \int_{0}^{u} f(t) d t
$$

cannot attain its maximum value in $\Omega$ unless it is a constant. 
We note that the only difference in demonstrating the nonnegativity of $L Q$ is that one need not add and subtract 1 as done in (2.5) since the term $c f^{2}$ occurs as a consequence of the placement of the parameter in $Q$. We also note that no specific lower derivative bound on $f$ is called for and that the coefficient of the nonlinear term in (2.8) is positive.

Now let us seek to weaken the requirement on $f^{\prime}$ in Theorem 1 , where $u$ is a $C^{3}$ solution of (1.4).

We define the function

$$
R(x)=\frac{u_{, k} u, k}{c(x)}+\gamma \int_{0}^{\varphi(u)} f(t) d t
$$

where $\gamma$ is a positive constant to be determined, $\varphi(u)$ is a function to be determined, and $c(x) \geq c_{0}>0$. We compute $R_{, i}$ and $R_{, i j}$ as before, where we use a prime to indicate differentiation with respect to the argument and we let $\bar{f}=\bar{f}(u)=$ $f(\varphi(u))$. Then as in (2.2) we form

$$
\begin{aligned}
L R= & 2 c^{-1} a^{i j} u_{, k j} u_{, k i}+2 c^{-1} a^{i j} u_{, k} u_{, k i j}-2 c^{-2} a^{i j} u_{, k} u_{, k i} c_{, j} \\
& -2 c^{-2} a^{i j} u_{, k} u_{, k j} c_{, i}-c^{-2} a^{i j} u_{, k} u_{, k} c_{, i j}+2 c^{-3} a^{i j} u_{, k} u_{, k} c_{, i} c_{, j} \\
& +\gamma \bar{f}^{\prime} \varphi^{\prime 2} a^{i j} u_{, j} u_{, i}+\gamma \bar{f} \varphi^{\prime \prime} a^{i j} u_{, j} u_{, i}+\gamma \bar{f} \varphi^{\prime} a^{i j} u_{, i j} \\
& +2 c^{-1} b^{i} u_{, k} u_{, k i}-c^{-2} b^{i} u_{, k} u_{, k} c_{, i}+\gamma \bar{f} \varphi^{\prime} b^{i} u_{, i} .
\end{aligned}
$$

Using (2.3) and collecting terms, we can write

$$
\begin{aligned}
L R= & 2 c^{-1} a^{i j} u_{, k j} u_{, k i}-2 c^{-1} a_{, k}^{i j} u_{, k} u_{, i j}-2 c^{-1} b_{, k}^{i} u_{, k} u_{, i} \\
& +2 c^{-1} f u_{, k} c_{, k}+2 f^{\prime} u_{, k} u_{, k}-4 c^{-2} a^{i j} u_{, k} u_{, k i} c_{, j}-c^{-2} u_{, k} u_{, k} L c \\
& +2 c^{-3} u_{, k} u_{, k} a^{i j} c_{, i} c_{, j}+\left(\gamma \bar{f}^{\prime} \varphi^{\prime 2}+\gamma \bar{f} \varphi^{\prime \prime}\right) a^{i j} u_{, j} u_{, i}+\gamma c \bar{f} \varphi^{\prime} f
\end{aligned}
$$

We note that we can again use (2.6) on the first, second, and sixth terms of (2.11). It only remains to use the tenth term to balance the "undesirable" fourth term of (2.11). We achieve this by assuming $\varphi^{\prime} \bar{f} f \geq \alpha^{2}>0$ and calculating

$$
\begin{aligned}
\gamma c \varphi^{\prime} \bar{f} f & +2 c^{-1} f u_{, k} c, k=(\gamma-1) c \varphi^{\prime} \bar{f} f+c \varphi^{\prime} \bar{f} f+2 c^{-1} f u_{, k} c, k \\
\geq & (\gamma-1) c \varphi^{\prime} \bar{f} f+c^{-1}\left[c^{2} \alpha^{2}+2 f u_{, k} c, k\right. \\
& \left.+(c \alpha)^{-2}\left(f u_{, k} c{ }_{, k}\right)^{2}-(c \alpha)^{-2}\left(f u_{, k} c, k\right)^{2}\right] \\
\geq & (\gamma-1) c \varphi^{\prime} \bar{f} f+c^{-1}\left[c \alpha+(c \alpha)^{-1} f u_{, k} c, k\right]^{2} \\
& -c^{-3} \alpha^{-2}|f|^{2}|\nabla c|^{2} u_{, k} u_{, k} .
\end{aligned}
$$

We now substitute (2.6) and (2.12) into (2.11) and collect terms. Assuming $\bar{f}^{\prime} \varphi^{\prime 2}+\bar{f} \varphi^{\prime \prime} \geq \beta>0$, this results in

$$
\begin{aligned}
& L R \geq\left\{\gamma \beta a^{i j}+\left(2 f^{\prime}-c^{-2} L c-c^{-3} \alpha^{-2}|f|^{2}|\nabla c|^{2}\right) \delta^{i j}-2 c^{-1} b_{, j}^{i}\right. \\
& -(2 c)^{-1}\left[A^{p q} a_{, j}^{p k} a_{, i}^{q k}+2 c^{-1} A^{p i} a_{, j}^{p k} a^{k m} c_{, m}\right. \\
& \left.\left.\quad+2 c^{-1} A^{j q} a^{k l} c_{, l} a_{, i}^{q k}+4 c^{-2} A^{j i} a^{k l} c_{, l} a^{k m} c, m\right]\right\} u_{, j} u_{, i} \\
& +2 c^{-3} u_{, k} u_{, k} a^{i j} c_{, i} c_{, j}+(\gamma-1) c \alpha^{2}+c^{-1}\left[c \alpha+(c \alpha)^{-1} f u_{, k} c_{, k}\right]^{2} .
\end{aligned}
$$

It is clear that the right side of (2.13) can be made nonnegative for a $\gamma$ chosen 
sufficiently large. Thus we have

THEOREM 3. If $u$ is a $C^{3}$ solution of (1.4), where the coefficients $a^{i j}, b^{i} \in$ $C^{1}(\bar{\Omega}), c \in C^{2}(\bar{\Omega})$, and $c(x) \geq c_{0}>0$ and the function $f \in C^{1}(R)$ is bounded and has its first derivative bounded below, and if there exists a function $\varphi$ such that

$$
\varphi^{\prime} \bar{f} f \geq \alpha^{2}>0, \quad \bar{f}^{\prime} \varphi^{\prime 2}+\bar{f} \varphi^{\prime \prime} \geq \beta>0,
$$

then there exists a positive constant $\gamma$ sufficiently large $(\gamma \geq 1)$ such that

$$
R(x)=\frac{u_{, k} u_{, k}}{c(x)}+\gamma \int_{0}^{\varphi(u)} f(t) d t
$$

cannot attain its maximum value in $\Omega$ unless it is a constant.

We note that nontrivial $f$ and $\varphi$ which satisfy the conditions in Theorem 3 for a solution which is bounded below are $f(u)=\arctan u^{2}+1$ and $\varphi(u)=e^{u}$. We also note that the first condition in (2.14) precludes Theorem 3 from strictly containing Theorem 1 inasmuch as it implies that $f(u) \neq 0$, which was not hypothesized in Theorem 1.

3. Bounds. For an application of Theorem 1, we consider the nonlinearity $f(u)=u^{3}+u$. We note that the integral term in $P(x)$ is nonnegative since $f(0)=0$ here. If we let $x_{0}$ be a point on $\partial \Omega$ at which $P(x)$ attains its maximum, then we obtain from $P(x) \leq P\left(x_{0}\right)$ that

$$
\frac{|\nabla u(x)|^{2}}{c(x)} \leq \frac{\left|\nabla u\left(x_{0}\right)\right|^{2}}{c\left(x_{0}\right)}+\gamma\left[\frac{1}{4} u^{4}\left(x_{0}\right)+\frac{1}{2} u^{2}\left(x_{0}\right)\right]
$$

for any $x \in \bar{D}$. Moreover, it follows from $P(x) \leq P\left(x_{0}\right)$ that

$$
|u(x)| \leq\left[\frac{2}{\gamma c_{0}}\left|\nabla u\left(x_{0}\right)\right|^{2}+\frac{1}{2} u^{4}\left(x_{0}\right)+u^{2}\left(x_{0}\right)\right]^{1 / 2}
$$

with this $f$. It is conceivable that a better solution estimate may be obtained by taking the fourth root, i.e.,

$$
|u(x)| \leq\left[\frac{4}{\gamma c_{0}}\left|\nabla u\left(x_{0}\right)\right|^{2}+u^{4}\left(x_{0}\right)+2 u^{2}\left(x_{0}\right)\right]^{1 / 4} .
$$

The above estimates apply to any nontrivial solution of (1.4) with $f$ as given. More generally, for any $f$ which satisfies the condition of Theorem 1 , if $f(0)=0$ and if one imposes homogeneous Dirichlet boundary conditions on $u$, then one can deduce the gradient estimate

$$
|\nabla u(x)| \leq K\left|\nabla u\left(x_{0}\right)\right|, \quad K^{2}=\left(1 / c_{0}\right) \max _{\bar{D}} c(x)
$$

where $x_{0}$ is some point on $\partial \Omega$ at which $P(x)$ attains its maximum.

For a physical application, we consider the single, irreversible, steady-state reaction studied in [1]. There the scalar problem for the concentration is formulated as

$$
\Delta u-k^{2} f(u)=0, \quad \text { in } \Omega, \quad u=1, x \in \partial \Omega, u \geq 0, x \in \Omega
$$


where $k^{2}$ is a positive constant and where $f$ has the properties $f:[0, \infty) \rightarrow[0, \infty)$, $f(0)=0, f(1)=1, f \in C^{1}, f>0, f$ monotone increasing on $(0, \infty)$. Clearly, $f(u)=\frac{1}{2}\left(u^{3}+u\right)$ has such properties.

In Lemma 3.1 of [1] a gradient bound, namely,

$$
|\operatorname{grad} u(x)|^{2} \leq 2 k^{2} \int_{m}^{u(x)} f(t) d t
$$

is given in terms of the value of $u$ at $x$ and $m=\min u(x)$ when the average curvature of the boundary is nonnegative. By Theorem 1 with $\gamma=1$ and without the restriction on the geometry, we obtain

$$
|\operatorname{grad} u(x)|^{2} \leq\left|\operatorname{grad} u\left(x_{0}\right)\right|^{2}+k^{2} \int_{0}^{1} f(t) d t,
$$

which could possibly be a better estimate. In fact, however, for a solution $u$ of (3.1) it can be shown (let $\gamma=0$ in Theorem 1) that $|\operatorname{grad} u(x)| \leq\left|\operatorname{grad} u\left(x_{0}\right)\right|$ for $x_{0}$ some point of $\partial \Omega$ since $|\operatorname{grad} u|^{2}$ takes its maximum on the boundary.

We have only briefly indicated how the principles here can be utilized to obtain estimates. We refer the reader to Sperb's text [7] for other applications of principles of the type presented here.

ACKNOWLEDGMENT. This work was accomplished while the author was a Visiting Guest Professor in the Mathematisches Institut I at the Universität Karlsruhe, West Germany. This opportunity was made available by a University of Tennessee Faculty Development Leave.

\section{REFERENCES}

1. C. Bandle, R. P. Sperb, and I. Stakgold, Diffusion and reaction with monotone kinetics, Nonlinear Anal. 8 (1984), 321-333.

2. Wen Duan Lu and Zhi Huo Yiang, Some results on maximum principles, Sichuan Daxue Xuebao (1981), 23-36. (Chinese)

3. L. E. Payne, Bounds for the maximum stress in the Saint Venant torsion problem, Indian J. Mech. Math. (1968), 51-59.

4. M. H. Protter and H. F. Weinberger, $A$ maximum principle and gradient bounds for linear elliptic equations, Indiana Univ. Math. J. 23 (1973), 239-249.

5. M. H. Protter, Gradient bounds for a class of second order elliptic equations, Contemp. Math. 11 (1982), 191-198.

6. R. P. Sperb, Maximum principles and nonlinear elliptic problems, J. Anal. Math. 35 (1979), 236-263.

7. __ Maximum principles and their applications, Academic Press, New York, 1981.

Department of Mathematics, University of Tennessee, Knoxville, TenNESSEE 37916 